\title{
A Fast Algorithm for Image Super-Resolution from Blurred Observations
}

\author{
Nirmal K. Bose, ${ }^{1}$ Michael K. Ng, ${ }^{2}$ and Andy C. Yau ${ }^{3}$ \\ ${ }^{1}$ Spatial and Temporal Signal Processing Center, Department of Electrical Engineering, The Pennsylvania State University, \\ University Park, PA 16802, USA \\ ${ }^{2}$ Department of Mathematics, Hong Kong Baptist University, Kowloon Tong, Hong Kong \\ ${ }^{3}$ Department of Mathematics, Faculty of Science, The University of Hong Kong, Pokfulam Road, Hong Kong, China
}

Received 1 December 2004; Revised 17 March 2005; Accepted 7 April 2005

\begin{abstract}
We study the problem of reconstruction of a high-resolution image from several blurred low-resolution image frames. The image frames consist of blurred, decimated, and noisy versions of a high-resolution image. The high-resolution image is modeled as a Markov random field (MRF), and a maximum a posteriori (MAP) estimation technique is used for the restoration. We show that with the periodic boundary condition, a high-resolution image can be restored efficiently by using fast Fourier transforms. We also apply the preconditioned conjugate gradient method to restore high-resolution images in the aperiodic boundary condition. Computer simulations are given to illustrate the effectiveness of the proposed approach.
\end{abstract}

Copyright (c) 2006 Hindawi Publishing Corporation. All rights reserved.

\section{INTRODUCTION}

Image sequence super-resolution refers to methods that increase spatial resolution by fusing information from a sequence of images (with partial overlap in successive elements or frames in, e.g., video), acquired in one or more of several possible ways. For brevity, in this context, either the term super-resolution or high resolution is used to refer to any algorithm which produces an increase in resolution from multiple low-resolution degraded images. At least, two nonidentical images are required to construct a higher-resolution version. The low-resolution frames may be displaced with respect to a reference frame (Landsat images, where there is a considerable distance between camera and scene), blurred (due to causes like optical aberration, relative motion between camera and object, atmospheric turbulence), rotated and scaled (due to video camera motion like zooming, panning, tilting), and, furthermore, those could be degraded by various types of noise like signal-independent or signal-dependent, multiplicative or additive.

Due to hardware cost, size, and fabrication complexity limitations, imaging systems like charge-coupled device (CCD) detector arrays often provide only multiple lowresolution degraded images. However, a high-resolution image is indispensable in applications including health diagnosis and monitoring, military surveillance, and terrain mapping by remote sensing. Other intriguing possibilities in- clude substituting expensive high-resolution instruments like scanning electron microscopes by their cruder, cheaper counterparts and then applying technical methods for increasing the resolution to that derivable with much more costly equipment. Resolution improvement by applying tools from digital signal processing technique has, therefore, been a topic of very great interest [1-15]. The attainment of image superresolution was based on the feasibility of reconstruction of two-dimensional bandlimited signals from nonuniform samples [16] arising from frames generated by microscanning, that is, subpixel shifts between successive frames, each of which provides a unique snapshot of a stationary scene.

In 1990, Kim et al. [8] proposed a weighted recursive least-squares algorithm based on sequential estimation theory in the Fourier transform or wavenumber domain for filtering and interpolating with the objective of constructing a high-resolution image from a registered sequence of undersampled, noisy, and blurred frames, displaced horizontally and vertically from each other (sufficient for Landsat-type-imaging). Kim and Su [17] incorporated explicitly the deblurring computation into the high-resolution image reconstruction process since separate deblurring of input frames would introduce the undesirable phase and high wavenumber distortions DFT of those frames. A discrete-cosine-transform (DCT) -based approach in the spatial domain with regularization, but without the recursive updating feature of [8], was recently considered in 
[11] and an optimization-theory-based approach with regularization was given in [5]. Bose et al. adapted a recursive total least-squares (TLSs) algorithm to tackle high-resolution reconstruction from low-resolution noisy sequences with displacement error during image registration [18]. A theory was advanced, through variance analysis, to assess the robustness of this TLS algorithm for image reconstruction [19]. Specifically, it was shown that with appropriate assumptions, the image reconstructed using the TLS algorithm has minimum variance with respect to all unbiased estimates. The most recent activities following the paper published in 1990 [8] in this vibrant area are summarized in some typical papers [20] (galactical image, X-ray image, satellite image of hurricane, city aerial image, CAT-scan of thoracic cavity), [21] (digital electron microscopy), [22] (super-resolution in magnetic resonance imaging) that serve to offer credence to the immense scope, diversity of applications, and the importance of the subject matter.

A different approach towards super-resolution from that in [8] was suggested in 1991 by Irani and Peleg [6], who used a rigid model instead of a translational model in the image registration process and then applied the iterative backprojection technique from computer-aided tomography. A summary of these and other research during the last decade is contained in a recent paper [23]. Mann and Picard [24] proposed the projective model in image registration because their images were acquired with a video camera. The projective model was subsequently used by Lertrattanapanich and Bose [25] for video mosaicing and high resolution.

An image acquisition system composed of an array of sensors, where each sensor has a subarray of sensing elements of suitable size, has recently been popular for increasing the spatial resolution with high signal-to-noise ratio beyond the performance bound of technologies that constrain the manufacture of imaging devices. The technique for reconstructing a high-resolution from data acquired by a prefabricated array of multisensors was advanced by Bose and Boo [1], and this work was further developed by applying total least squares to account for error not only in observation but also due to error in estimation of parameters modeling the data [26]. The method of projection onto convex sets (POCS) has been applied to the problem of reconstruction of a highresolution image from a sequence of undersampled degraded frames. Sauer and Allebach applied the POCS algorithm to this problem subject to the blur-free assumption [27]. Stark and Oskoui [13] applied POCS in the blurred but noise-free case. Patti et al. [14] formulated a POCS algorithm to compute an estimate from low-resolution images obtained by either scanning or rotating an image with respect to the CCD image acquisition sensor array or mounting the image on a moving platform [5].

Nonuniform spacing of the data samples in frames is at the heart of super-resolution, and this may be coupled with presence of data dropouts or missing data. In early research, Ur and Gross [28] discussed a nonuniform interpolation scheme based on the generalized sampling theorem of Papoulis and Brown [28] while Jacquemod et al. [7] proposed interpolation followed by least-squares restoration. The wavelet basis offers considerable promise in the fast inter- polation of unevenly spaced data. Motivated by the promise of wavelets, a couple of papers on wavelet super-resolution have appeared [29-31]. These papers use only first generation wavelets and also do not subscribe to the need for selecting the mother wavelet to optimize performance.

In this paper, we focus on the problem of reconstructing a high-resolution image from several blurred low-resolution image frames. The image frames consist of decimated, blurred, and noisy versions of the high-resolution image $[32,33]$. The high-resolution image is modeled as a Markov random field (MRF), and a maximum a posteriori (MAP) estimation technique is used for the restoration. We propose to use the preconditioned conjugate gradient method [34] instead to optimize the MAP objective function. We show that with the periodic boundary condition, the high-resolution image can be restored efficiently by using fast Fourier transforms (FFTs). In particular, an $n$-by- $n$ high-resolution image can be restored by using two-dimensional FFTs in $O\left(n^{2} \log n\right)$ operations. We remark that such approach has been proposed and studied by Bose and Boo [1] for high-resolution image reconstruction. Here, we consider a more general blurring matrix in the image reconstruction. By using our results, we construct a preconditioner for solving the linear system arising from the optimization of the MAP objective function when other boundary conditions are considered. Both theoretical and numerical results show that the preconditioned conjugate gradient method converges very quickly, and also the high-resolution image can be restored efficiently by the proposed method.

In our proposed method, we have assumed that the blur kernel is known. However, when the blur kernel is not known, the problem of multiframe blind deconvolution occurs. A promising approach to multiframe blur identification was proposed by Biggs and Andrews [35]. Their iterative blind deconvolution method uses the popular Richardson-Lucy algorithm. Further generalization of the result in [35] to include not only multiple blur identifications but also support estimation of blurs (the blur supports were assumed to be either known a priori or determined by trial and error) has recently been completed in [36] and used in blind super-resolution. The problem of super-resolved depth recovery from defocused images by blur parameter estimation in the task of image super-resolution has been reported in [37].

The outline of the paper is as follows. In Section 2, we briefly give a mathematical formulation of the problem. In Section 3, we study how to use fast Fourier transforms to restore high-resolution images efficiently. Finally, numerical results and concluding remarks are given in Section 4.

\section{MATHEMATICAL FORMULATION}

In this section, we give an introduction to the mathematical model for the high-resolution image restoration. Let us consider the low-resolution sensor plane with $m$-by- $m$ sensors elements. Suppose that the downsampling parameter is $q$ in both the horizontal and vertical directions. Then the highresolution image is of size $q m$-by- $q m$. The high-resolution image $Z$ has intensity values $Z=\left[z_{i, j}\right]$, for $i=0, \ldots, q m-1$, $j=0, \ldots, q m-1$. The high-resolution image is first blurred 
by a different, but known linear space-invariant blurring function. They have the following relation:

$$
\hat{z}_{i, j}=h(i, j) * z_{i, j}
$$

where $h(i, j)$ is a blurring function and “ $*$ " denotes the discrete convolution.

The low-resolution image $Y$ has intensity values $Y=$ $\left[y_{i, j}\right]$, for $i=0, \ldots, m-1, j=0, \ldots, m-1$. The relationship between $Y$ and $\hat{Z}$ can be written as follows:

$$
y_{i, j}=\frac{1}{q^{2}} \sum_{l=i m+1}^{(i+1) m} \sum_{k=j m+1}^{(j+1) m} \hat{z}_{l, k} .
$$

We consider the low-resolution intensity to be the average of the blurred high-resolution intensities over a neighborhood of $q^{2}$ pixels.

Let $\mathbf{z}$ be a vector of size $q^{2} m^{2}$-by- 1 containing the intensity of the high-resolution image $Z$ in a chosen lexicographical order. Let $\mathbf{y}_{i}$ be the $m^{2}$-by-1 lexicographically ordered vector containing the intensity value of the blurred, decimated, and noisy image $Y_{i}$. Then, the matrix form can be written as (far-field imaging)

$$
\mathbf{y}_{i}=\mathbf{D H}_{i} \mathbf{z}+\mathbf{n}_{i}
$$

where $\mathbf{D}$ is a (real-valued) decimation matrix of size $m^{2}$-by$q^{2} m^{2}, \mathbf{H}_{i}$ is a real-valued blurring matrix (due to atmospheric turbulence, e.g.) of size $q^{2} m^{2}$-by- $q^{2} m^{2}$, and $\mathbf{n}_{i}$ is an $m^{2}$-by1 noise vector. The decimation matrix $\mathbf{D}$ has the form $(q$ nonzero elements, each of value $1 / q^{2}$ in each row)

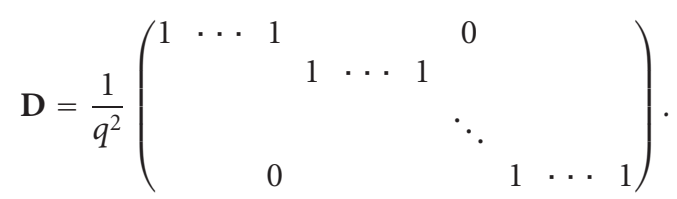

The noise vector $\mathbf{n}_{i}$ is assumed to be zero-mean independent and identically distributed of the form

$$
P\left(\mathbf{n}_{i}\right)=\frac{1}{(2 \pi)^{m^{2} / 2} \sigma^{m^{2}}} e^{-\left(1 / 2 \sigma^{2}\right) \mathbf{n}_{i}^{T} \mathbf{n}_{i}} .
$$

By using a MAP estimation technique [33], we find that the cost function of this model is given by

$$
\min _{\mathbf{z}}\left\{\sum_{i=1}^{p}\left\|\mathbf{y}_{i}-\mathbf{D H}_{i} \mathbf{z}\right\|_{2}^{2}+\alpha\|\mathbf{L z}\|_{2}^{2}\right\},
$$

where $p$ is the number of observed low-resolution images, $\alpha$ is a regularization parameter, and $\mathbf{L}$ is the first-order finitedifference matrix, and $\mathbf{L}^{T} \mathbf{L}$ is the discrete Laplacian matrix. In the above formulation, the noise variance term is absorbed in the regularization parameter $\alpha$. The minimization of the cost function (6) is equivalent to the solving of the following linear system:

$$
\left(\sum_{i=1}^{p} \mathbf{H}_{i}^{T} \mathbf{D}^{T} \mathbf{D} \mathbf{H}_{i}+\alpha \mathbf{L}^{T} \mathbf{L}\right) \mathbf{z}=\sum_{i=1}^{p} \mathbf{H}_{i}^{T} \mathbf{D}^{T} \mathbf{y}_{i}
$$

In the next section, we will discuss the coefficient matrix of the linear system (7) and suggest an algorithm to solve the above system efficiently.

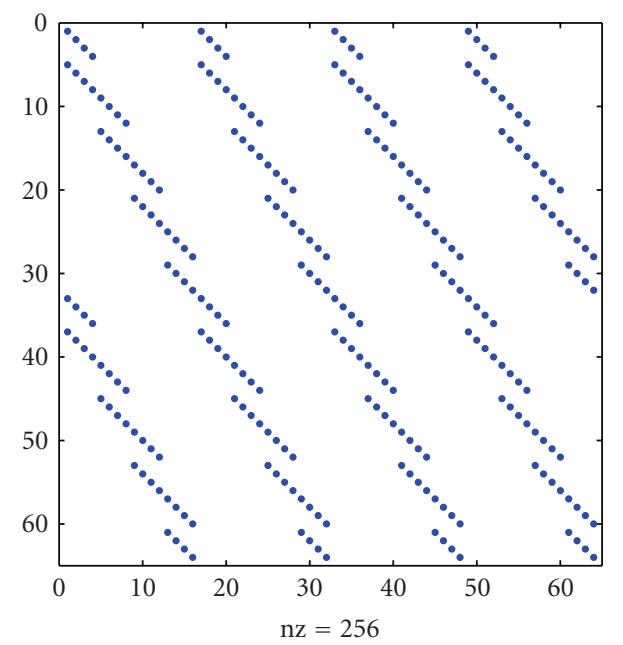

Figure 1: Example of Theorem 1 for $m=4$ and $q=2$.

\section{ANALYSIS FOR PERIODIC BLURRING MATRICES}

In this section, we discuss the linear system (7) for periodic blurring matrices, that is, the blurring matrix $\mathbf{H}_{i}$ under the periodic boundary condition. Then the linear system (7) becomes

$$
\left(\sum_{i=1}^{p} \mathbf{C}_{i}^{T} \mathbf{D}^{T} \mathbf{D} \mathbf{C}_{i}+\alpha \mathbf{L}_{c}^{T} \mathbf{L}_{c}\right) \mathbf{z}=\sum_{i=1}^{p} \mathbf{C}_{i}^{T} \mathbf{D}^{T} \mathbf{y}_{i}
$$

where $\mathrm{C}_{i}$ is a block-circulant-circulant-block (BCCB) blurring matrix and $\mathbf{L}_{c}^{T} \mathbf{L}_{c}$ is a Laplacian matrix in BCCB structure.

Notice that $\mathbf{C}_{i}^{T} \mathbf{D}^{T} \mathbf{D} \mathbf{C}_{i}$ is singular for all $i$ since $\mathbf{D C} \mathbf{C}_{i}$ is not of full rank, and $\mathbf{L}_{c}^{T} \mathbf{L}_{c}$ is positive semidefinite but it has only one zero eigenvalue. The corresponding eigenvector is equal to $\mathbf{1}=(1, \ldots, 1)^{T}$, that is,

$$
\left(\sum_{i=1}^{p} \mathbf{C}_{i}^{T} \mathbf{D}^{T} \mathbf{D C}_{i}+\alpha \mathbf{L}_{c}^{T} \mathbf{L}_{c}\right) \mathbf{1}=\left(\sum_{i=1}^{p} \mathbf{C}_{i}^{T} \mathbf{D}^{T} \mathbf{D} \mathbf{C}_{i}\right) \mathbf{1} \neq \mathbf{0}
$$

This shows that the coefficient matrix $\sum_{i=1}^{p} \mathbf{C}_{i}^{T} \mathbf{D}^{T} \mathbf{D} \mathbf{C}_{i}+$ $\alpha \mathbf{L}_{c}^{T} \mathbf{L}_{c}$ is nonsingular. Therefore, the system (8) can be uniquely solved and the high-resolution image can be restored.

\subsection{Decomposition of coefficient matrix}

In this subsection, we discuss the coefficient matrix of the linear system (8). Similar to the previous case, the coefficient matrix consists of two parts: the blurred down/upsampling matrix $\sum_{i=1}^{p} \mathbf{C}_{i}^{T} \mathbf{D}^{T} \mathbf{D} \mathbf{C}_{i}$ and the regularization matrix $\alpha \mathbf{L}_{c}^{T} \mathbf{L}_{c}$.

Since the regularization matrix $\alpha \mathbf{L}_{c}^{T} \mathbf{L}_{c}$ is a BCCB matrix, we can use the tensor product $\mathbf{R}_{2}=\mathbf{F}_{m q} \otimes \mathbf{F}_{m q}$ (where $\mathbf{F}_{m q}$ is the complex-valued discrete Fourier transform matrix of size $m q$-by-mq) to diagonalize $\mathbf{L}_{c}^{T} \mathbf{L}_{c}$,

$$
\Lambda_{\mathbf{L}_{c}}=\mathbf{R}_{2} \mathbf{L}_{c}^{T} \mathbf{L}_{c} \mathbf{R}_{2}^{*} .
$$




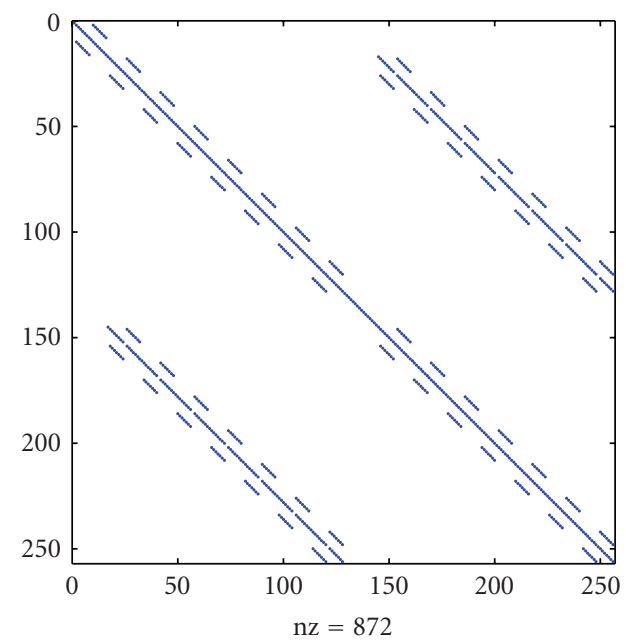

FIgURE 2: The structure of the matrix $\mathbf{R S R}^{*}+\alpha \boldsymbol{\Lambda}_{\mathbf{L}_{c}}$.

Note that the asterisk superscript denotes complex conjugate transpose of the matrix.

The first part $\sum_{i=1}^{p} \mathbf{C}_{i}^{T} \mathbf{D}^{T} \mathbf{D} \mathbf{C}_{i}$ of the coefficient matrix has a multilevel structure so that it cannot be diagonalized directly by $\mathbf{R}_{2}=\mathbf{F}_{m q} \otimes \mathbf{F}_{m q}$. However, we can permute this matrix into the circulant-block matrix

$$
\mathbf{E}=\mathbf{P}_{1}\left(\sum_{i=1}^{p} \mathbf{C}_{i}^{T} \mathbf{D}^{T} \mathbf{D} \mathbf{C}_{i}\right) \mathbf{P}_{1}^{T}=\left(\begin{array}{cccc}
\mathbf{A}_{1,1} & \mathbf{A}_{1,2} & \ldots & \mathbf{A}_{1, q} \\
\mathbf{A}_{2,1} & \mathbf{A}_{2,2} & \ldots & \mathbf{A}_{2, q} \\
\vdots & \vdots & \ddots & \vdots \\
\mathbf{A}_{q, 1} & \mathbf{A}_{q, 2} & \ldots & \mathbf{A}_{q, q}
\end{array}\right),
$$

where $\mathbf{P}_{1}$ is a permutation matrix and $\mathbf{A}_{i, j}$ is of size $q m^{2}$-by$q m^{2}$. Each $\mathbf{A}_{i, j}$ can be partitioned into $q$-by- $q$ BCCB matrices, that is,

$$
\mathbf{A}_{i, j}=\left(\begin{array}{cccc}
\mathbf{B}_{1,1} & \mathbf{B}_{1,2} & \ldots & \mathbf{B}_{1, q} \\
\mathbf{B}_{2,1} & \mathbf{B}_{2,2} & \ldots & \mathbf{B}_{2, q} \\
\vdots & \vdots & \ddots & \vdots \\
\mathbf{B}_{q, 1} & \mathbf{B}_{q, 2} & \ldots & \mathbf{B}_{q, q}
\end{array}\right)
$$

where $\mathbf{B}_{i, j}$ is of size $m^{2}$-by- $m^{2}$. It follows that the matrix $\mathbf{E}$ in (11) can be block-diagonalized by the tensor product of the complex-valued discrete Fourier transform matrix $\mathbf{R}_{1}=$ $\mathbf{I}_{q^{2}} \otimes \mathbf{F}_{m} \otimes \mathbf{F}_{m}$. Then, we have the block-diagonal matrix $\mathbf{S}=$ $\mathbf{R}_{1} \mathbf{E R}_{1}^{*}$. The system (7) becomes

$$
\left(\mathbf{R S R}^{*}+\alpha \boldsymbol{\Lambda}_{\mathbf{L}_{c}}\right) \mathbf{R}_{2} \mathbf{Z}=\mathbf{R}_{2} \sum_{i=1}^{p} \mathbf{C}_{i}^{T} \mathbf{D}^{T} \mathbf{y}_{i}
$$

where $\mathbf{R}=\mathbf{R}_{2}\left(\mathbf{R}_{1} \mathbf{P}_{1}\right)^{*}$. Next, we will show that the matrix $\mathbf{R}$ is a sparse matrix.

Theorem 1. Let $\mathbf{F}_{n}$ be the n-by-n discrete Fourier matrix and let $\mathbf{I}_{n}$ be the identity matrix of size $n-b y-n$. Then,

$$
\mathbf{R}_{2} \mathbf{P}_{1}^{*} \mathbf{R}_{1}^{*} \begin{cases}\neq 0, & a-l=0(\bmod m), x-y=0(\bmod m), \\ =0 & \text { otherwise, }\end{cases}
$$

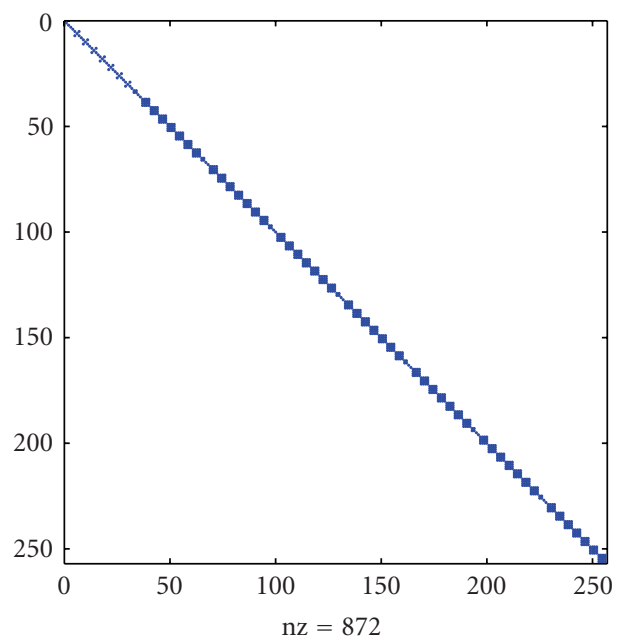

FIGURE 3: The structure of the matrix $\mathbf{R S R}^{*}+\alpha \boldsymbol{\Lambda}_{\mathbf{L}_{c}}$ after permutation.

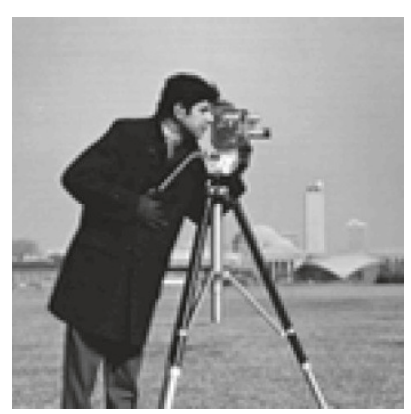

(a)

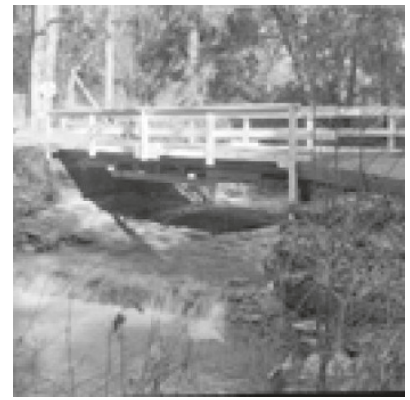

(b)
Figure 4: (a) The cameraman and (b) the bridge.

where $\mathbf{R}_{1}=\mathbf{I}_{q^{2}} \otimes \mathbf{F}_{m} \otimes \mathbf{F}_{m}, \mathbf{R}_{2}=\mathbf{F}_{m q} \otimes \mathbf{F}_{m q}$, and $\mathbf{P}_{1}$ is a permutation matrix. For those nonzero entries, they are given by

$$
m^{2} e^{(-2 \pi i[(a-1)(k-1)+(x-1)(t-1)]) / m q} .
$$

Here $x$ and $y$ are the row and column indices of the matrix $\mathbf{R}_{2} \mathbf{P}_{1}^{*} \mathbf{R}_{1}^{*}$, respectively, with $l=r(b, m+1)+1$, with $b=$ $y \bmod m^{2}$ for $y \neq m^{2}$ otherwise $b=m^{2}, a=r(x, q m+1)+1$, $k=r\left(y, m^{2}+1\right)+1, t=k \bmod q$ for $k=n q$ otherwise $t=q$, and $r(c, d)$ denotes the integral part of $c / d$.

The proof of this theorem is given in the appendix. This theorem shows that $\mathbf{R}$ is a sparse matrix. Figure 1 demonstrates the sparsity of the matrix $\mathbf{R}$ when $m=4$ and $q=2$.

The dot represents the nonzero entries in the matrix $\mathbf{R}$. By using Theorem 1, the nonzero entries of the matrix $\mathbf{R}$ can be precomputed with a low computational cost.

According to Theorem 1, the structure of $\mathbf{R}$ can be described as follows. The matrix $\mathbf{R}$ can be considered as a $q$ by- $q^{2}$ block matrix and the size of each block matrix is $q m^{2}$ by- $m$. Each block matrix has the same structure. In particular, each block matrix can be again considered as an $m$-by- $m$ 


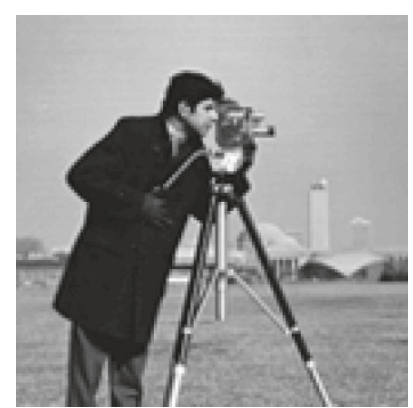

(a)

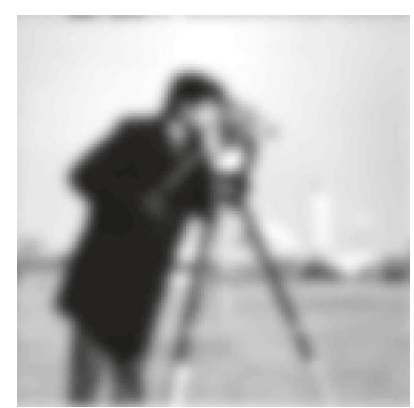

(b)

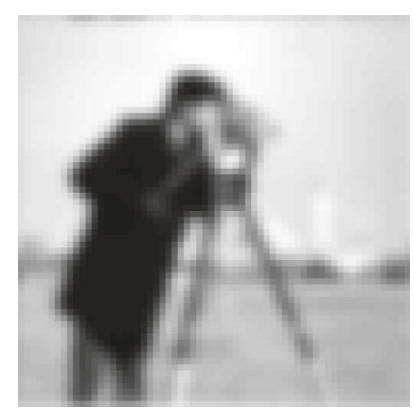

(c)

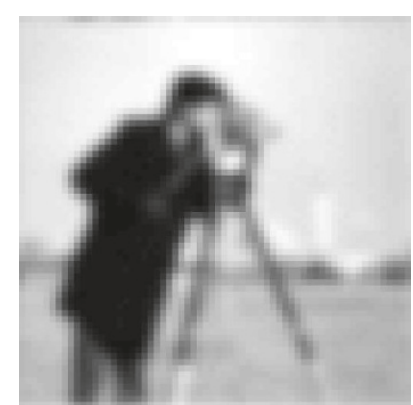

(d)

Figure 5: The formation of observed low-resolution image: (a) the original image; (b) the blurred image; (c) the decimated and blurred image; (d) the decimated and blurred noisy image.

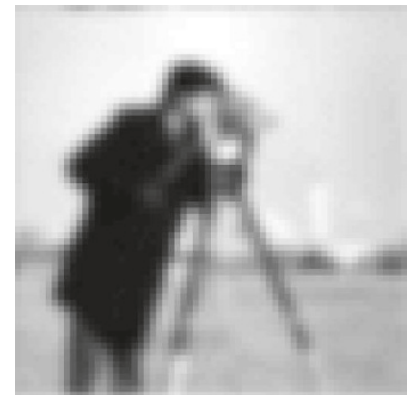

(a)

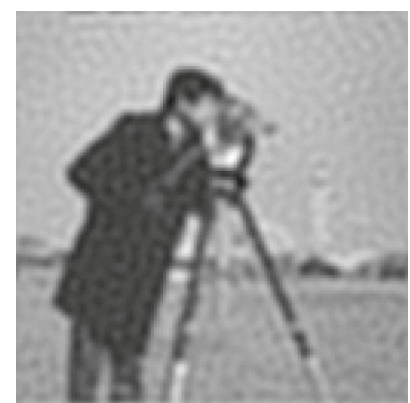

(b)

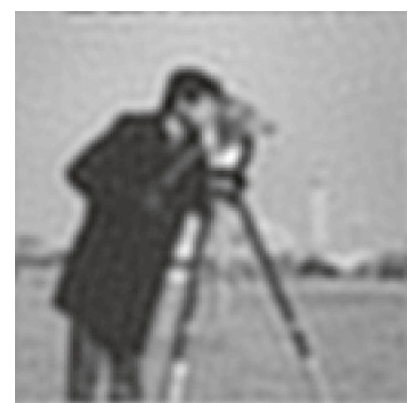

(c)

FIgURE 6: (a) The blurred low-resolution image with $\gamma=5$ and noise level $=40 \mathrm{~dB}$, (b) and its restored images with $\alpha=0.1$ (relative error $=0.11903$ ), and (c) $\alpha=0.5$ (relative error $=0.12252$ ).

block matrix and the size of each block is $q m$-by- $m$. In this level, all the blocks are just zero matrices except the main diagonal blocks. Such diagonal block matrices are $q$-by-1 block with block-diagonal matrix of size $m$-by- $m$. According to this nice structure, there are at most $m$ nonzero entries in each row and each column of $\mathbf{R}$, and it implies that $\mathbf{R}$ is a sparse matrix.

\subsection{The computational algorithm}

By using Theorem 1 and the fact that $\mathbf{S}$ is a block-diagonal matrix, it is clear that the matrix $\mathbf{R}^{*} \mathbf{S R}$ is sparse, and therefore the matrix $\mathbf{R}^{*} \mathbf{S R}+\alpha \boldsymbol{\Lambda}_{\mathbf{L}_{c}}$ is also sparse. In Figure 2, we present a structure of the resultant matrix for $m=8$ and $q=2$.

We find that the resultant matrix can be partitioned into $q$-by- $q$ block matrices of size $q m^{2}$-by- $q m^{2}$. Due to the structure of $\mathbf{R}$, each block matrix is a banded matrix with bandwidth $(q-1) m+1$. Then, we can permute those nonzero entries of the resultant matrix such that the permuted matrix becomes a block-diagonal matrix. Each block matrix is of size $q^{2}$-by- $q^{2}$. Therefore, the linear system (8) can be expressed as a block-diagonalized system of decoupled subsystems. Thus, linear equations can be computed by solving a set of $m^{2}$ decoupled $q^{2}$-by- $q^{2}$ matrix equations. We show the resultant matrix in Figure 3 after permutation of Figure 2. We summarize the algorithm as follows:

(i) input $\left\{Y_{i}\right\},\left\{\mathbf{C}_{i}\right\}, q$, and $\alpha$;

(ii) compute $S$ and $\Lambda_{\mathbf{L}_{c}}$;

(iii) compute $\mathbf{R}$ by using Theorem 1 ;

(iv) compute $\mathbf{R S R}^{*}+\alpha \boldsymbol{\Lambda}_{\mathbf{L}_{c}}$;

(v) compute the inverse of $\mathbf{R S R}^{*}+\alpha \boldsymbol{\Lambda}_{\mathbf{L}_{c}}$;

(vi) output the reconstructed high-resolution image $Z$.

Table 1 shows the computational cost of each matrix computation of the above algorithm.

We note that $m \gg q$, therefore for an $q m$-by- $q m$ highresolution image, the complexity of the proposed algorithm is $O\left(q^{2} m^{2} \log q m\right)$ operations.

\section{APERIODIC BLURRING MATRICES}

For the aperiodic boundary condition, we denote that $\mathbf{T}_{i}$ is the block-Toeplitz-Toeplitz-block matrix, and denote $\mathbf{L}_{e}^{T} \mathbf{L}_{e}$ to be the discrete Laplacian matrix with the zero boundary condition. Then, the system (7) becomes

$$
\left(\sum_{i=1}^{p} \mathbf{T}_{i}^{T} \mathbf{D}^{T} \mathbf{D} \mathbf{T}_{i}+\alpha \mathbf{L}_{e}^{T} \mathbf{L}_{e}\right) \mathbf{z}=\sum_{i=1}^{p} \mathbf{T}_{i}^{T} \mathbf{D}^{T} \mathbf{y}_{i}
$$




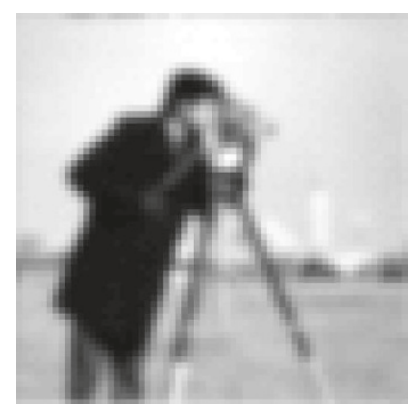

(a)

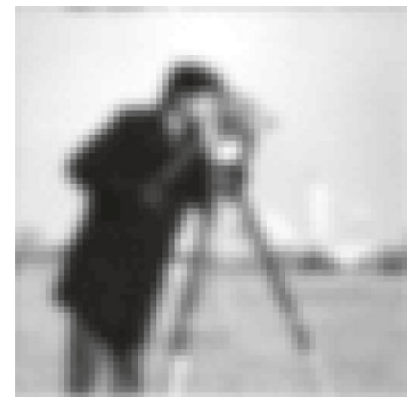

(d)

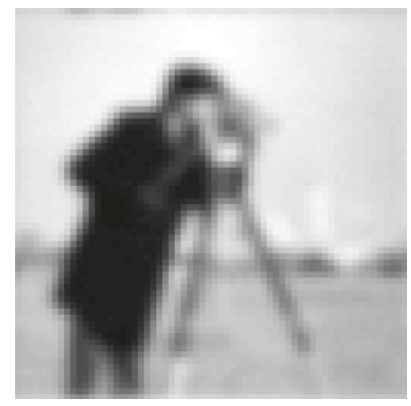

(g)

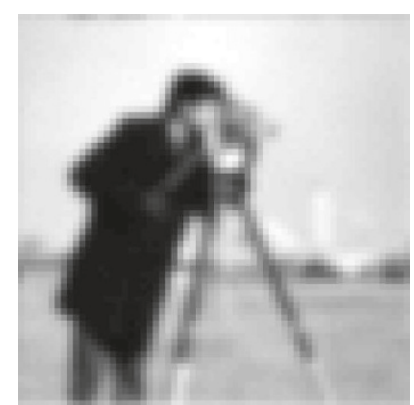

(b)

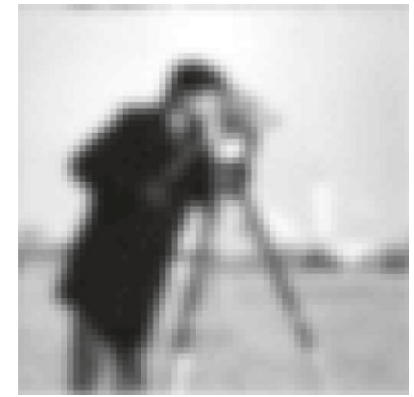

(e)

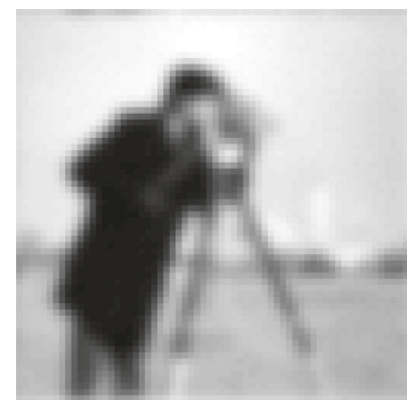

(h)

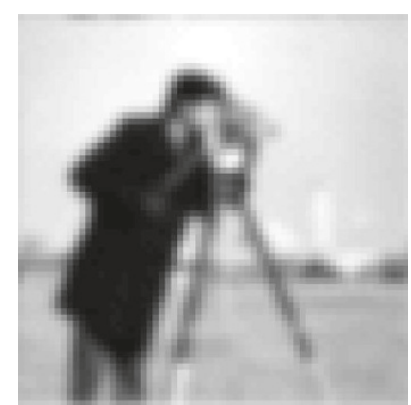

(c)

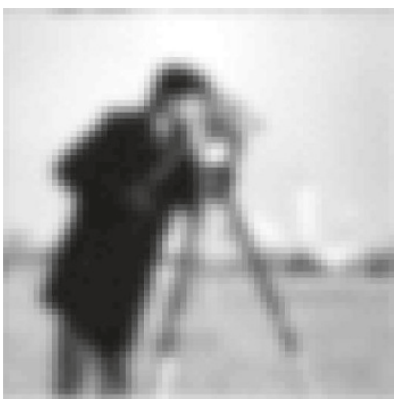

(f)

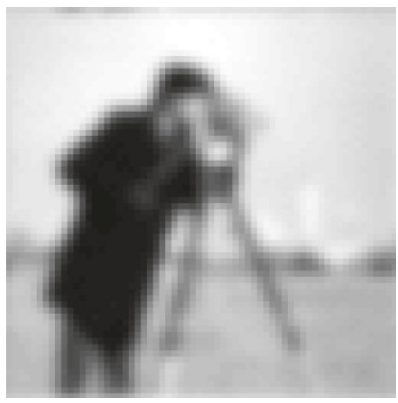

(i)

FIGURE 7: Nine blurred low-resolution images with $\gamma=3.4,3.8,4.2,4.6,5,5.4,5.8,6.2,6.6$ and noise level $=40 \mathrm{~dB}$.

In this case, we employ a circulant matrix $\mathbf{C}_{i}$ to approximate the Toeplitz matrix $\mathbf{T}_{i}$. Similarly, we use $\mathbf{L}_{c}^{T} \mathbf{L}_{c}$ to be the discrete Laplacian matrix with the periodic boundary condition to approximate $\mathbf{L}_{e}^{T} \mathbf{L}_{e}$. Then, the preconditioner is given by

$$
\left(\sum_{i=1}^{p} \mathbf{C}_{i}^{T} \mathbf{D}^{T} \mathbf{D} \mathbf{C}_{i}+\alpha \mathbf{L}_{c}^{T} \mathbf{L}_{c}\right) \mathbf{z}=\sum_{i=1}^{p} \mathbf{C}_{i}^{T} \mathbf{D}^{T} \mathbf{y}_{i}
$$

which is exactly the linear system in (8). Therefore, we can use the same decomposition as before. Also, as the preconditioned matrix is symmetric positive definite, we can apply the preconditioned conjugate gradient method with the above preconditioner to solve the system (16) efficiently.

The problem of approximation of a block-Toeplitz matrix by a block-circulant matrix has been analyzed in [38]. The equidistribution property of multidimensional sequences is used to show that sequences of BTTB (block-
Toeplitz-Toeplitz-blocks) and BCCB (block-circulant-circulant-blocks) matrices are asymptotically equivalent in a certain sense.

\section{NUMERICAL RESULTS}

In this section, we will discuss numerical results. A 128-by128 image is taken to be the original high-resolution image, and the desired high-resolution image is restored from several 64-by-64 noisy, blurred, and undersampled images, that is, we take the downsampling parameter $q=2$. Two original 128-by-128 images "cameraman" and "bridge" are shown in Figure 4. by

We assume the blur to be a Gaussian blur which is given

$$
H_{i, j}=e^{-D^{2}(i, j) / 2 \gamma}
$$


TABLE 1: The computation cost of the proposed algorithm.

\begin{tabular}{c|cc}
\hline Computed matrix & Size & Operations \\
\hline $\boldsymbol{S}$ & $q^{2} m^{2} \times 1$ & $O\left(q^{2} m^{2} \log (m)\right)$ \\
$\boldsymbol{\Lambda}_{\mathbf{L}_{c}}$ & $q^{2} m^{2} \times 1$ & $O\left(q^{2} m^{2} \log (q m)\right)$ \\
$\mathbf{R S R}^{*}$ & $q^{2} m^{2} \times q^{2} m^{2}$ & $O\left(q^{4} m^{2}\right)$ \\
$\mathbf{R S R}^{*}+\alpha \boldsymbol{\Lambda}_{\mathbf{L}_{c}}$ & $q^{2} m^{2} \times q^{2} m^{2}$ & $O(q m)$ \\
$\left(\mathbf{R S R}^{*}+\alpha \boldsymbol{\Lambda}_{\mathbf{L}_{c}}\right)^{-1}$ & $q^{2} m^{2} \times q^{2} m^{2}$ & $O\left(m^{2} q^{6}\right)$ \\
\hline \multirow{2}{*}{ Total } & - & $O\left(q^{4} m^{2}+q^{6} m^{2}+q^{2} m^{2} \log (m)\right.$ \\
& & $\left.+q^{2} m^{2} \log (q m)\right)$ \\
\hline
\end{tabular}

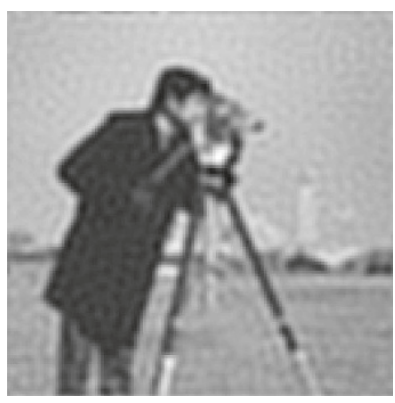

(a)

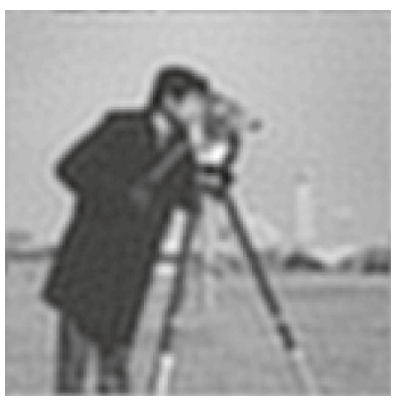

(b)
Figure 8: (a) The restored images with $\alpha=0.02$ (relative error $=$ $0.10536)$ and (b) $\alpha=0.08$ (relative error $=0.11042$ ).

The size of the blurring kernel for this model is 29, that is, 29 pixels of the image will be affected by the blurring matrix. All blurred images are simulated by using FFT multiplication.

\subsection{Periodic blurring matrices}

We first discuss the results for the periodic case. Figure 5 shows the high-resolution image $\mathbf{z}$, the blurred image $\mathbf{H}_{i} \mathbf{z}$, the decimated and blurred image $\mathbf{D H}_{i} \mathbf{z}$, and the decimated and blurred noisy image $\mathbf{D H}_{i} \mathbf{z}+\mathbf{n}_{i}$. Figure 6 shows that the super-resolution image is obtained by the single observed image. The optimal regularization parameter is $\alpha=0.1$ and its relative error is 0.11903 . We also show another restored image with $\alpha=0.5$ for comparison and its relative error is 0.12252 . The optimal regularization parameter $\alpha$ is chosen such that it minimizes the relative error of the reconstructed high-resolution image $\mathbf{z}_{r}(\alpha)$ to the original image $\mathbf{z}$, that is, it minimizes

$$
\frac{\left\|\mathbf{z}-\mathbf{z}_{r}(\alpha)\right\|_{2}}{\|\mathbf{z}\|_{2}}
$$

In Figures 7 and 8, nine low-resolution images and their corresponding restored images are shown. The optimal regularization parameter $\alpha=0.02$, and the relative error is 0.10536 . Another restored image with $\alpha=0.08$ is shown for the comparison and the relative error is 0.11042 . Table 2 shows further results for periodic blurring matrices. The results show that if we input more low-resolution images, we can get more accurate high-resolution image and lower optimal regularization parameter $\alpha$ as more information is provided.

\subsection{Aperiodic blurring matrices}

We have discussed in Section 4 employing the preconditioned conjugate gradient method with circulant preconditioners to solve (16). Here, we show the results for aperiodic blurring matrices.

Figure 9 shows the restored image from a single image. The optimal regularization parameter is $\alpha=0.09$ and the relative error is 0.12448 . The numbers of conjugate gradient iterations with and without using preconditioner are 96 and 177, respectively. Another restored image with $\alpha=0.15$ and its relative error is 0.12535 is shown. The numbers of conjugate gradient iterations with and without using preconditioners are 75 and 145, respectively. Figures 10 and 11 show other examples where the super-resolution image is obtained by seven low-resolution images. The optimal regularization parameter is $\alpha=0.02$ and the relative error is 0.11289 . The numbers of conjugate gradient iterations with and without using preconditioner are 194 and 301. Another restored image with $\alpha=0.1$ and its relative error is 0.11838 is shown. The numbers of conjugate gradient iterations with and without using preconditioners are 89 and 166. We find that the use of circulant preconditioner can speed up the conjugate gradient method, and therefore the high-resolution restored image can be obtained more efficiently.

\section{THE COMPARISON BETWEEN TWO SUPER-RESOLUTION IMAGING MODELS}

In this section, we compare the model in (3) with another super-resolution imaging model [33] (near-field imaging):

$$
\mathbf{y}_{i}=\mathbf{H}_{i}{ }_{i} \mathbf{D z}+\mathbf{n}_{i},
$$

where $\mathbf{D}$ is a decimation matrix of size $m^{2}$-by- $q^{2} m^{2}, \mathbf{H}^{\prime}{ }_{i}$ is a blurring matrix (due to, say, optical aberration) of size $m^{2}$-by- $m^{2}$, and $\mathbf{n}_{i}$ is an $m^{2}$-by- 1 noise vector. The highresolution image can be reconstructed by the minimization of the following objective function:

$$
\min _{\mathbf{z}}\left\{\sum_{i=1}^{p}\left\|\mathbf{y}_{i}-\mathbf{H}_{i}^{\prime} \mathbf{D z}\right\|_{2}^{2}+\alpha\|\mathbf{L z}\|_{2}^{2}\right\} .
$$




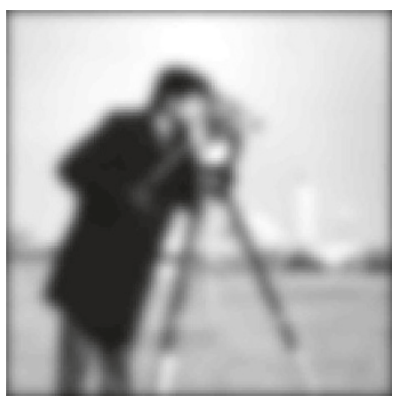

(a)

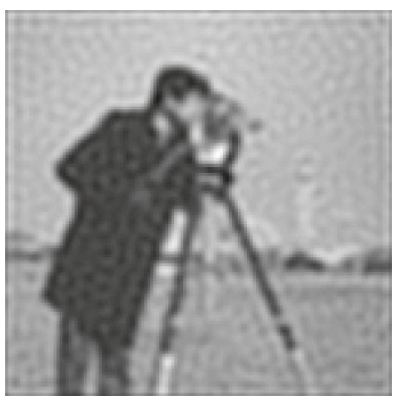

(b)

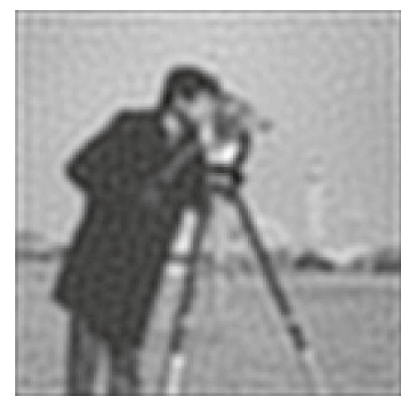

(c)

FIgURE 9: (a) The low-resolution image with $\gamma=5$ and noise level $=40 \mathrm{~dB}$, (b) its corresponding restored images with $\alpha=0.09$ (relative error $=0.12448$ and PCG iterations $=96$ ), and $(c) \alpha=0.15$ (relative error $=0.12535$ and PCG iterations $=75$ ).

TABLE 2: The optimal regularization parameters and the corresponding relative errors.

\begin{tabular}{|c|c|c|c|c|c|c|}
\hline \multirow{3}{*}{ Number of input images } & \multicolumn{6}{|c|}{ Noise level } \\
\hline & \multicolumn{2}{|c|}{$30 \mathrm{~dB}$} & \multicolumn{2}{|c|}{$40 \mathrm{~dB}$} & \multicolumn{2}{|c|}{$50 \mathrm{~dB}$} \\
\hline & $\begin{array}{c}\text { Optimal } \\
\alpha\end{array}$ & $\begin{array}{c}\text { Relative } \\
\text { error }\end{array}$ & $\begin{array}{c}\text { Optimal } \\
\alpha\end{array}$ & $\begin{array}{c}\text { Relative } \\
\text { error }\end{array}$ & $\begin{array}{c}\text { Optimal } \\
\alpha\end{array}$ & $\begin{array}{c}\text { Relative } \\
\text { error }\end{array}$ \\
\hline 1 & 1.2 & 0.13300 & 0.1 & 0.11903 & 0.01 & 0.10666 \\
\hline 3 & 0.4 & 0.12577 & 0.04 & 0.11263 & 0.005 & 0.10119 \\
\hline 5 & 0.2 & 0.12204 & 0.02 & 0.10943 & 0.003 & 0.09815 \\
\hline 7 & 0.2 & 0.12000 & 0.02 & 0.10710 & 0.003 & 0.09620 \\
\hline 9 & 0.1 & 0.11856 & 0.02 & 0.10536 & 0.003 & 0.09481 \\
\hline
\end{tabular}

TABLE 3: The comparison of both models in the periodic case.

\begin{tabular}{c|cc|cc}
\hline \multirow{2}{*}{ Number of input images } & \multicolumn{2}{|c|}{ Model in (3) } & \multicolumn{2}{c}{ Model in (20) } \\
\cline { 2 - 5 } & $\begin{array}{c}\text { Optimal } \\
\alpha\end{array}$ & Relative error & $\begin{array}{c}\text { Optimal } \\
\alpha\end{array}$ & Relative error \\
\hline 1 & 1.1 & 0.1836 & 0.060 & 0.1834 \\
2 & 0.3 & 0.1493 & 0.010 & 0.1478 \\
3 & 0.2 & 0.1507 & 0.008 & 0.1491 \\
4 & 0.1 & 0.1503 & 0.005 & 0.1487 \\
5 & 0.1 & 0.1531 & 0.005 & 0.1509 \\
\hline
\end{tabular}

We remark that under the same blurring function, the sizes of blurring matrices $\mathbf{H}_{i}^{\prime}$ and $\mathbf{H}_{i}$ in these two models are different, and the numbers of pixels affected by these two blurring matrices are also different.

Table 3 shows the results for these two imaging models. We find that the relative errors using the model in (3) are slightly larger than those using the model in (20). Figures 12 and 13 show five observed low-resolution images in these two models with the same blurring functions. Figure 14 shows the restored images for these two models. The optimal regularization parameters are $\alpha=0.005$ and $\alpha=0.1$ for (20) and (3), respectively. Their relative errors are 0.1531 and
0.1509 for (3) and (20), respectively. We see that both superresolution imaging models give about the same relative errors. Visually, the quality of both restored images is about the same. This observation is also true for other cases in the table.

In the summary, we have studied super-resolution restoration from several decimated, blurred, and noisy image frames. Also, we have developed algorithms to restore the high-resolution image. Experimental results demonstrated that the method is quite effective and efficient. Model for both near-field and far-field image blur still remains to be tackled-a difficult problem because of noncommutativity of relevant operators in the models. 


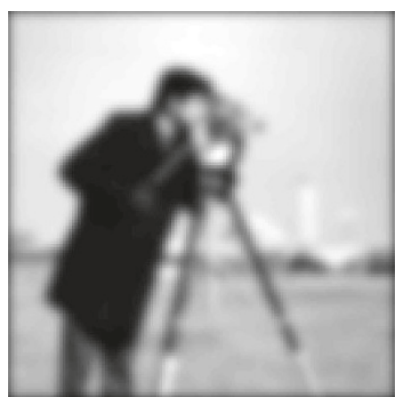

(a)

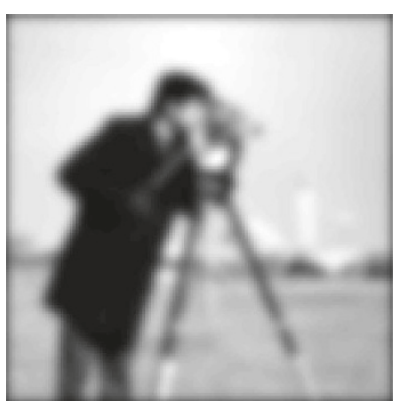

(b)

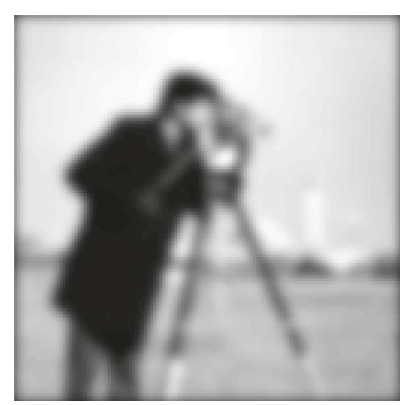

(c)

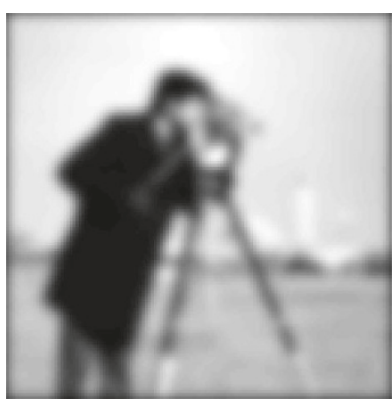

(d)

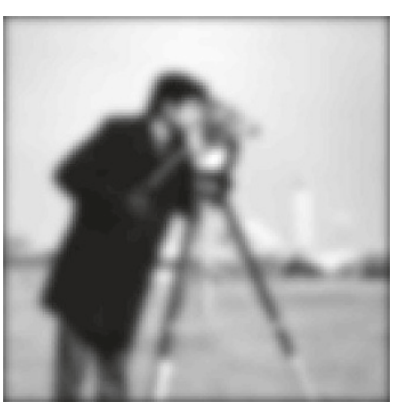

(e)

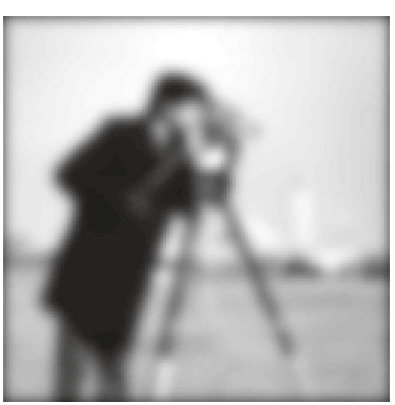

(f)

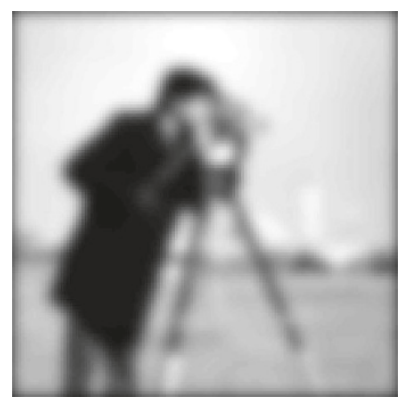

(g)

FIGURE 10: Seven blurred images with $\gamma=3.8,4.2,4.6,5,5.4,5.8,6.2$ and noise level $=40 \mathrm{~dB}$.

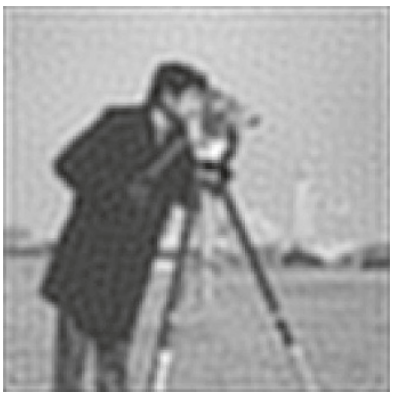

(a)

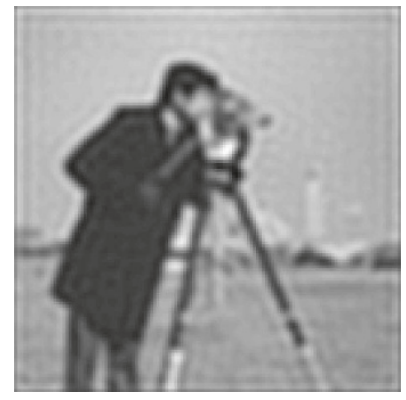

(b)

FIGURE 11: (a) The restored images with $\alpha=0.02$ (relative error $=0.11289$ and PCG iterations $=194)$ and (b) $\alpha=0.1$ (relative error $=0.11838$ and PCG iterations $=89$ ).

\section{APPENDIX}

Proof of Theorem 1. We can partition $\mathbf{F}_{m}^{*}$ as follows:

$$
\begin{aligned}
\mathbf{F}_{m}^{*} & =\left(\begin{array}{cccc}
1 & 1 & \cdots & 1 \\
1 & e^{2 \pi i / m} & \cdots & e^{2 \pi i(m-1) / m} \\
\vdots & \vdots & \ddots & \vdots \\
1 & e^{2 \pi i(m-1) / m} & \cdots & e^{2 \pi i(m-1)(m-1) / m}
\end{array}\right) \\
& =\left(\begin{array}{cccc}
f_{1,1} & f_{1,2} & \cdots & f_{1, m} \\
f_{2,1} & f_{2,2} & \cdots & f_{2, m} \\
\vdots & \vdots & \ddots & \vdots \\
f_{m, 1} & f_{m, 2} & \cdots & f_{m, m}
\end{array}\right)
\end{aligned}
$$

where $f_{j, k}=e^{2 \pi i(j-1)(k-1) / m}$. Then the matrix $\mathbf{R}_{1}^{*}=\left(\mathbf{I}_{q^{2}} \otimes\right.$ $\left.\mathbf{F}_{m} \otimes \mathbf{F}_{m}\right)^{*}$ is equal to

$$
\left(\begin{array}{cccc}
\mathbf{F}_{m}^{*} \otimes \mathbf{F}_{m}^{*} & 0 & \cdots & 0 \\
0 & \mathbf{F}_{m}^{*} \otimes \mathbf{F}_{m}^{*} & \cdots & 0 \\
0 & 0 & \ddots & 0 \\
0 & 0 & \cdots & \mathbf{F}_{m}^{*} \otimes \mathbf{F}_{m}^{*}
\end{array}\right) .
$$

After the permutation, the matrix becomes

$$
\begin{aligned}
\mathbf{P}^{*} \times \mathbf{R}_{1}^{*} & =\left(\begin{array}{cccc}
\mathbf{H}_{1,1} & \mathbf{H}_{1,2} & \cdots & \mathbf{H}_{1, q^{2}} \\
\mathbf{H}_{2,1} & \mathbf{H}_{2,2} & \cdots & \mathbf{H}_{2, q^{2}} \\
\vdots & \vdots & \ddots & \\
\mathbf{H}_{m q, 1} & \mathbf{H}_{m q, 2} & \cdots & \mathbf{H}_{m q, q^{2}}
\end{array}\right) \\
& =\left(\begin{array}{llll}
\mathbf{Q}_{1} & \mathbf{Q}_{2} & \cdots & \mathbf{Q}_{q}
\end{array}\right),
\end{aligned}
$$




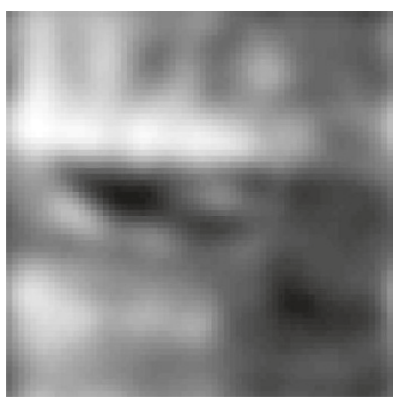

(a)

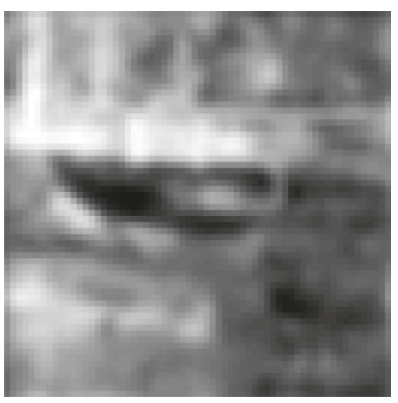

(b)

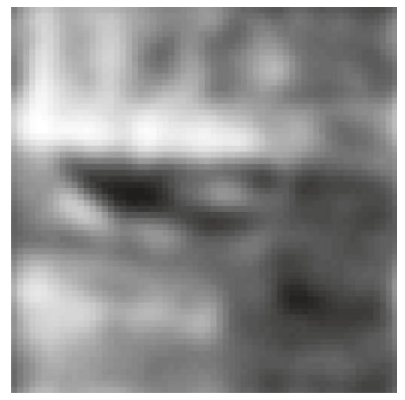

(c)

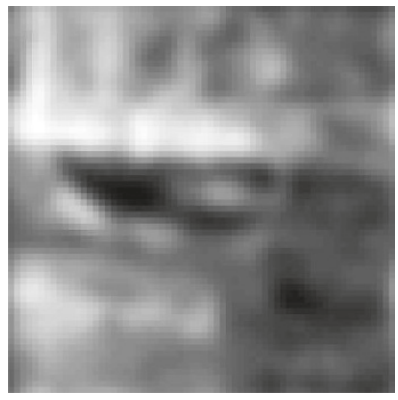

(d)

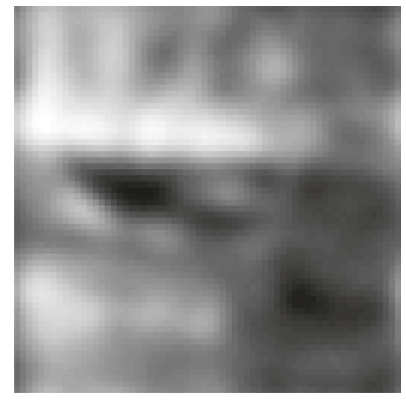

(e)

FIGURE 12: Five blurred images for the model in (3), with $\gamma=20,5,13,10,18$ and noise level $=40 \mathrm{~dB}$.

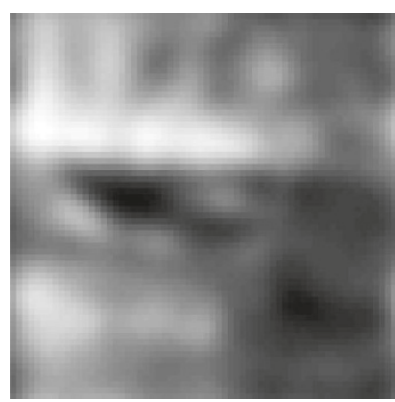

(a)

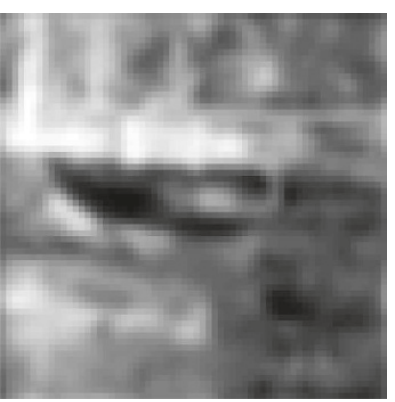

(b)

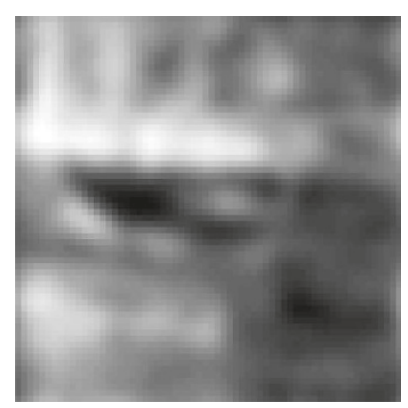

(c)

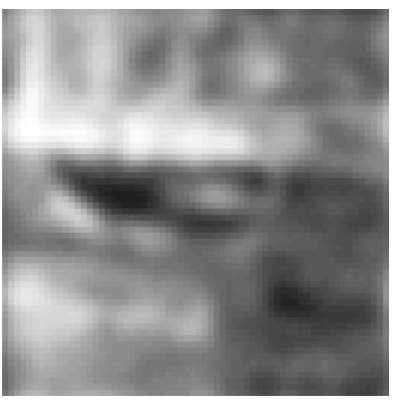

(d)

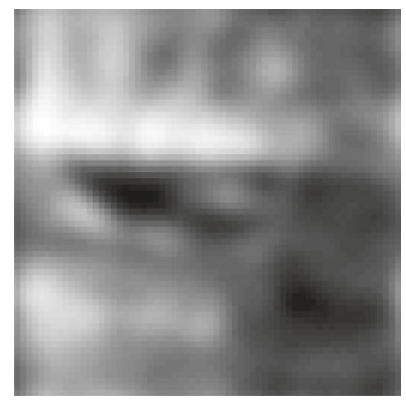

(e)

FIGURE 13: Five blurred images for the model in (20), with $\gamma=20,5,13,10,18$ and noise level $=40 \mathrm{~dB}$. 


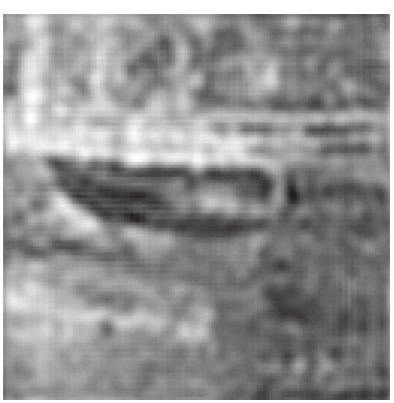

(a)

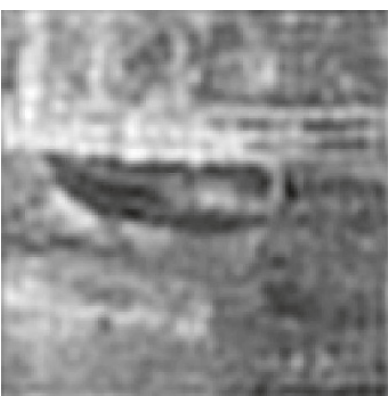

(b)

FIGURE 14: (a) The restored images with $\alpha=0.1$ (relative error $=0.15311$ ) for the model in (3) and (b) $\alpha=0.005$ (relative error $=0.15090$ ) for the model in (20).

where $\mathbf{Q}_{\mathbf{k}}$ is a matrix of size $q^{2} m^{2} \times q m^{2}$ for $k=1, \ldots, q$, that is,

$$
\begin{aligned}
& \left(\begin{array}{ccc}
\mathbf{H}_{1,(k-1) q+1} & \cdots & \mathbf{H}_{1, k q} \\
\mathbf{H}_{2,(k-1) q+1} & \cdots & \mathbf{H}_{2, k q} \\
\vdots & \ddots & \vdots \\
\mathbf{H}_{m q,(k-1) q+1} & \cdots & \mathbf{H}_{m q, k q}
\end{array}\right),
\end{aligned}
$$

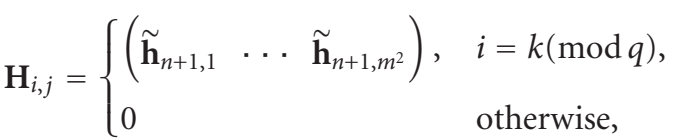

where $n$ is an integral part of $i /(q+1)$ and $\tilde{\mathbf{h}}_{n+1, y}=$ $e^{2 n \pi i(l-1) / m}\left(\mathbf{h}_{1} \mathbf{h}_{2} \cdots \mathbf{h}_{m q}\right)^{T}$ with

$$
\begin{gathered}
\mathbf{h}_{i}= \begin{cases}f_{\hat{n}+1, y}, & i=t(\bmod q), \\
0 & \text { otherwise, }\end{cases} \\
t= \begin{cases}j \bmod q, & j \neq \tilde{n} q, \\
q, & j=\tilde{n} q,\end{cases}
\end{gathered}
$$

for $\hat{n}$ is an integral part of $i /(q+1)$ and $n=0, \ldots, m-1, l$ is an integral part of $(y /(m+1)+1)$.

For $\mathbf{F}_{m q}$, we can partition as follows:

$$
\begin{aligned}
\mathbf{F}_{m q} & =\left(\begin{array}{cccc}
1 & 1 & \cdots & 1 \\
1 & e^{-2 \pi i / m q} & \cdots & e^{-2 \pi i(m q-1) / m q} \\
\vdots & \vdots & \ddots & \vdots \\
1 & e^{-2 \pi i(m q-1) / m q} & \cdots & e^{-2 \pi i(m q-1)(m q-1) / m q}
\end{array}\right) \\
& =\left(\begin{array}{llll}
\mathbf{g}_{1} & \mathbf{g}_{2} & \cdots & \mathbf{g}_{m q}
\end{array}\right)^{T},
\end{aligned}
$$

where $\mathbf{g}_{i}$ is the $i$ th row vector of $\mathbf{F}_{m q}$.
We note that $\mathbf{R}_{2}=\mathbf{F}_{m q} \otimes \mathbf{F}_{m q}$ and it becomes

$$
\begin{aligned}
& \mathbf{F}_{m q} \otimes \mathbf{F}_{m q}
\end{aligned}
$$

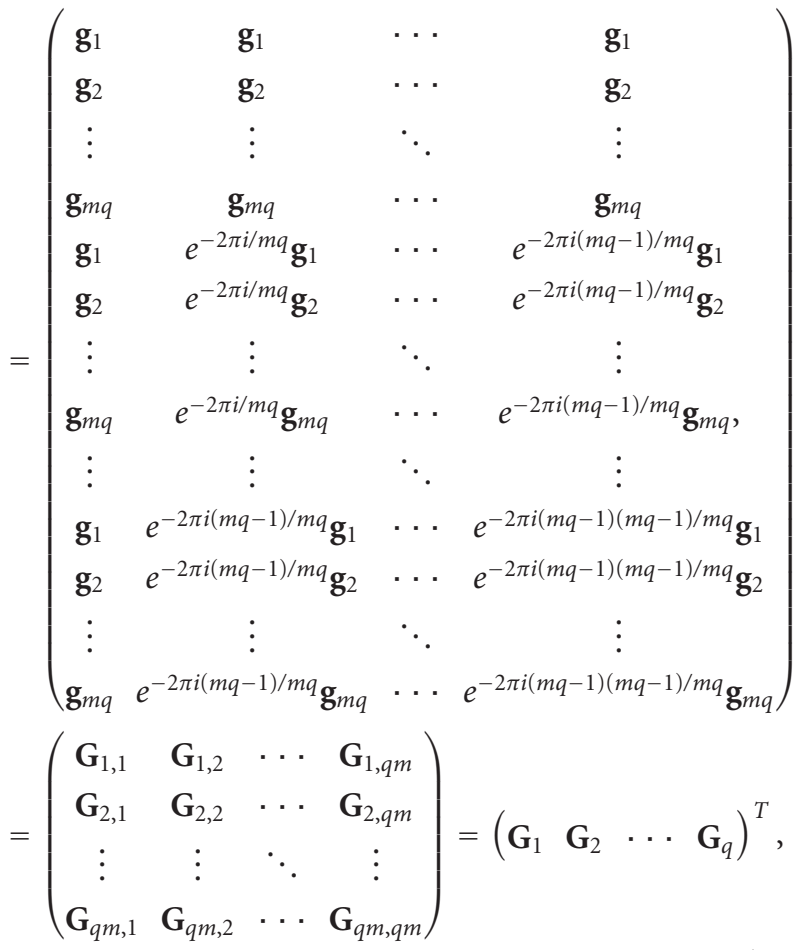

where

$$
\begin{aligned}
\mathbf{G}_{\mathbf{i}} & =\left(\begin{array}{cccc}
\mathbf{G}_{(i-1) m+1,1} & \mathbf{G}_{(i-1) m+1,2} & \cdots & \mathbf{G}_{(i-1) m+1, q m} \\
\mathbf{G}_{(i-1) m+2,1} & \mathbf{G}_{(i-1) m+2,2} & \cdots & \mathbf{G}_{(i-1) m+2, q m} \\
\vdots & \vdots & \ddots & \vdots \\
\mathbf{G}_{i m, 1} & \mathbf{G}_{i m, 2} & \cdots & \mathbf{G}_{i m, q m}
\end{array}\right) \\
& \in C^{m^{2} q \times m^{2} q^{2}}, \\
\mathbf{G}_{j, k} & =e^{-2 \pi i(j-1)(k-1) / m q}\left(\begin{array}{llll}
\mathbf{g}_{1} & \mathbf{g}_{2} & \cdots & \mathbf{g}_{m q}
\end{array}\right)^{T} \\
& \in C^{m q \times m q} .
\end{aligned}
$$


Then, we have

$$
\begin{aligned}
& \mathbf{R}_{2} \times \mathbf{P}^{*} \times \mathbf{R}_{1}^{*}=\left(\begin{array}{llll}
\mathbf{G}_{1} & \mathbf{G}_{2} & \cdots & \mathbf{G}_{\mathbf{q}}
\end{array}\right)^{T}\left(\begin{array}{llll}
\mathbf{Q}_{1} & \mathbf{Q}_{2} & \cdots & \mathbf{Q}_{q}
\end{array}\right)=\left(\begin{array}{cccc}
\mathbf{G}_{1} \mathbf{Q}_{1} & \mathbf{G}_{1} \mathbf{Q}_{2} & \cdots & \mathbf{G}_{1} \mathbf{Q}_{q} \\
\mathbf{G}_{2} \mathbf{Q}_{1} & \mathbf{G}_{2} \mathbf{Q}_{2} & \cdots & \mathbf{G}_{2} \mathbf{Q}_{q} \\
\vdots & \vdots & \ddots & \vdots \\
\mathbf{G}_{q} \mathbf{Q}_{1} & \mathbf{G}_{q} \mathbf{Q}_{2} & \cdots & \mathbf{G}_{q} \mathbf{Q}_{q}
\end{array}\right) \\
& \mathbf{G}_{j} \mathbf{Q}_{k}=\left(\begin{array}{cccc}
\mathbf{G}_{(j-1) m+1,1} & \mathbf{G}_{(j-1) m+1,2} & \cdots & \mathbf{G}_{(j-1) m+1, q m} \\
\mathbf{G}_{(j-1) m+2,1} & \mathbf{G}_{(j-1) m+2,2} & \cdots & \mathbf{G}_{(j-1) m+2, q m} \\
\vdots & \vdots & \ddots & \vdots \\
\mathbf{G}_{j m, 1} & \mathbf{G}_{j m, 2} & \cdots & \mathbf{G}_{j m, q m}
\end{array}\right) \times\left(\begin{array}{ccc}
\mathbf{H}_{1,(k-1) q+1} & \cdots & \mathbf{H}_{1, k q} \\
\mathbf{H}_{2,(k-1) q+1} & \cdots & \mathbf{H}_{2, k q} \\
\vdots & \ddots & \vdots \\
\mathbf{H}_{m q,(k-1) q+1} & \cdots & \mathbf{H}_{m q, k q}
\end{array}\right) \\
& =\left(\begin{array}{ccc}
\sum_{i=1}^{m} \mathbf{G}_{(j-1) m+1,(i-1) q+k} \mathbf{H}_{(i-1) q+k,(k-1) q+1} & \cdots & \sum_{i=1}^{m} \mathbf{G}_{(j-1) m+1,(i-1) q+k} \mathbf{H}_{(i-1) q+k, k q} \\
\sum_{i=1}^{m} \mathbf{G}_{(j-1) m+2,(i-1) q+k} \mathbf{H}_{(i-1) q+k,(k-1) q+1} & \cdots & \sum_{i=1}^{m} \mathbf{G}_{(j-1) m+2,(i-1) q+k} \mathbf{H}_{(i-1) q+k, k q} \\
\vdots & \ddots & \vdots \\
\sum_{i=1}^{m} \mathbf{G}_{j m,(i-1) q+k} \mathbf{H}_{(i-1) q+k,(k-1) q+1} & \cdots & \sum_{i=1}^{m} \mathbf{G}_{j m,(i-1) q+k} \mathbf{H}_{(i-1) q+k, k q}
\end{array}\right), \\
& \sum_{i=1}^{m} \mathbf{G}_{j m+s,(i-1) q+k} \mathbf{H}_{(i-1) q+k,(k-1) q+t}=\mathbf{G}_{j m+s, k} \mathbf{H}_{k,(k-1) q+t}+\mathbf{G}_{j m+s, q+k} \mathbf{H}_{q+k,(k-1) q+t}+\cdots+\mathbf{G}_{j m+s,(m-1) q+k} \mathbf{H}_{(m-1) q+k,(k-1) q+t},
\end{aligned}
$$

where $s=1, \ldots, m$ and $t=1, \ldots, q$.

Denoting $a=j m+s$ and $c=(k-1) q+t$ for $j, k=1, \ldots, q$, we have

$$
\begin{aligned}
\mathbf{G}_{a, u q+k} \mathbf{H}_{u q+k, c} & e^{-2 \pi i(a-1)(u q+k-1) / m q}\left(\begin{array}{llll}
\mathbf{g}_{1} & \mathbf{g}_{2} & \cdots & \mathbf{g}_{m q}
\end{array}\right)^{T} \\
& \times\left(\begin{array}{llll}
\widetilde{\mathbf{h}}_{u+1,1} & \widetilde{\mathbf{h}}_{u+1,2} & \cdots & \widetilde{\mathbf{h}}_{u+1, m^{2}}
\end{array}\right) \\
= & e^{-2 \pi i(a-1)(u q+k-1) / m q} \\
& \times\left(\begin{array}{cccc}
\mathbf{g}_{1} \widetilde{\mathbf{h}}_{u+1,1} & \mathbf{g}_{1} \widetilde{\mathbf{h}}_{u+1,2} & \cdots & \mathbf{g}_{1} \widetilde{\mathbf{h}}_{u+1, m^{2}} \\
\mathbf{g}_{2} \widetilde{\mathbf{h}}_{u+1,1} & \mathbf{g}_{2} \widetilde{\mathbf{h}}_{u+1,2} & \cdots & \mathbf{g}_{2} \widetilde{\mathbf{h}}_{u+1, m^{2}} \\
\vdots & \vdots & \ddots & \vdots \\
\mathbf{g}_{m q} \widetilde{\mathbf{h}}_{u+1,1} & \mathbf{g}_{m q} \widetilde{\mathbf{h}}_{u+1,2} & \cdots & \mathbf{g}_{m q} \widetilde{\mathbf{h}}_{u+1, m^{2}}
\end{array}\right) .
\end{aligned}
$$

For each entry, we have

$$
\begin{aligned}
& e^{-2 \pi i(a-1)(u q+k-1) / m q} \mathbf{g}_{x} \widetilde{\mathbf{h}}_{u+1, y} \\
& =e^{-2 \pi i(a-1)(u q+k-1) / m q} \\
& \times\left(\begin{array}{llll}
1 & e^{-2 \pi i(x-1) / m q} & \cdots & e^{-2 \pi i(x-1)(m q-1) / m q}
\end{array}\right),
\end{aligned}
$$$$
e^{2 u \pi i(l-1) / m}\left(\begin{array}{llll}
\mathbf{h}_{1} & \mathbf{h}_{2} & \cdots & \mathbf{h}_{m q}
\end{array}\right)^{T}
$$$$
=e^{-2 \pi i(a-1)(u q+k-1) / m q} e^{2 u \pi i(l-1) / m},
$$$$
\left(e^{-2 \pi i(x-1)(t-1) / m q} \mathbf{h}_{t}+e^{-2 \pi i(x-1)(q+t-1) / m q} \mathbf{h}_{q+t}\right.
$$$$
\left.+\cdots+e^{-2 \pi i(x-1)((m-1) q+t-1) / m q} \mathbf{h}_{(m-1) q+t}\right)
$$$$
=e^{-2 \pi i(a-1)(u q+k-1) / m q} e^{2 u \pi i(l-1) / m} e^{-2 \pi i(x-1)(t-1) / m q},
$$$$
\left(1+e^{-2 \pi i(x-1) q / m q} e^{2 \pi i(y-1) / m}\right.
$$$$
\left.+\cdots+e^{-2 \pi i(x-1)(m-1) q / m q} e^{2 \pi i(m-1)(y-1) / m}\right)
$$$$
=e^{-2 \pi i[u q(a-l)+(a-1)(k-1)+(x-1)(t-1)] / m q}
$$$$
\times\left(1+e^{2 \pi i(x-y) / m}+\cdots+e^{2 \pi i(m-1)(x-y) / m}\right)
$$$$
=e^{-2 \pi i u(a-l) / m} e^{-2 \pi i[(a-1)(k-1)+(x-1)(t-1)] / m q}
$$$$
\times\left(1+\omega+\cdots+\omega^{m-1}\right), \quad \omega=e^{2 \pi i(x-y) / m},
$$$$
=e^{-2 \pi i u(a-l) / m} e^{-2 \pi i[(a-1)(k-1)+(x-1)(t-1)] / m q} \sum_{v=0}^{m-1} \omega^{v}
$$$$
=m e^{-2 \pi i u(a-l) / m} e^{-2 \pi i[(a-1)(k-1)+(x-1)(t-1)] / m q}
$$

for $x-y=0(\bmod m)$. 
Adding the same entry for different matrix $\mathbf{G}_{a, u q+k} \mathbf{H}_{u q+k, c}$, we get

$$
\begin{aligned}
& \sum_{u=0}^{m-1} m e^{-2 \pi u i(s-l) / m} e^{-2 \pi i[(a-1)(k-1)+(x-1)(t-1)] / m q} \\
& =m e^{-2 \pi i[(a-1)(k-1)+(x-1)(t-1)] / m q} \sum_{u=0}^{m-1} e^{-2 \pi u i(s-l) / m} \\
& =m e^{-2 \pi i[(a-1)(k-1)+(x-1)(t-1)] / m q} \sum_{u=0}^{m-1} \alpha^{u} \\
& \quad\left(\text { where } \alpha=e^{-2 \pi i(a-l) / m}\right) \\
& =m^{2} e^{-2 \pi i[(a-1)(k-1)+(x-1)(t-1)] / m q}
\end{aligned}
$$$$
\text { for } a-l=0 \bmod m \text {. }
$$

The result follows.

\section{ACKNOWLEDGMENTS}

This research is supported by Army Research Office Grant DAAD 19-03-1-0261 and National Science Foundation Grant CCF-0429481. This research is supported in part by RGC Grants no. HKU 7130/02P, no. 7046/03P, no. 7035/04P, and no. 10205775 .

\section{REFERENCES}

[1] N. K. Bose and K. J. Boo, "High-resolution image reconstruction with multisensors," International Journal of Imaging Systems and Technology, vol. 9, no. 4, pp. 294-304, 1998.

[2] M. Elad and A. Feuer, "Restoration of a single super-resolution image from several blurred, noisy and undersampled measured images," IEEE Transactions on Image Processing, vol. 6, no. 12, pp. 1646-1658, 1997.

[3] M. Elad and A. Feuer, "Super-resolution restoration of an image sequence: adaptive filtering approach," IEEE Transactions on Image Processing, vol. 8, no. 3, pp. 387-395, 1999.

[4] J. C. Gillette, T. M. Stadtmiller, and R. C. Hardie, "Aliasing reduction in staring infrared imagers utilizing subpixel techniques," Optical Engineering, vol. 34, no. 11, pp. 3130-3137, 1995.

[5] R. C. Hardie, K. J. Barnard, J. G. Bognar, E. E. Armstrong, and E. A. Watson, "High resolution image reconstruction from a sequence of rotated and translated frames and its application to an infrared imaging system," Optical Engineering, vol. 37, no. 1, pp. 247-260, 1998.

[6] M. Irani and S. Peleg, "Improving resolution by image registration," CVGIP: Graphical Models and Image Processing, vol. 53, no. 3, pp. 231-239, 1991.

[7] G. Jacquemod, C. Odet, and R. Goutte, "Image resolution enhancement using subpixel camera displacement," Signal Processing, vol. 26, no. 1, pp. 139-146, 1992.

[8] S. P. Kim, N. K. Bose, and H. M. Valenzuela, "Recursive reconstruction of high resolution image from noisy undersampled multiframes," IEEE Transactions on Acoustics, Speech, and Signal Processing, vol. 38, no. 6, pp. 1013-1027, 1990.

[9] T. Komatsu, K. Aizawa, T. Igarashi, and T. Saito, "Signalprocessing based method for acquiring very high resolution images with multiple cameras and its theoretical analysis," IEE Proceedings. I, Communications, Speech and Vision, vol. 140, no. 1, pp. 19-24, 1993.

[10] A. J. Patti, M. I. Sezan, and A. Murat Tekalp, "Super-resolution video reconstruction with arbitrary sampling lattices and nonzero aperture time," IEEE Transactions on Image Processing, vol. 6, no. 8, pp. 1064-1076, 1997.

[11] S. H. Rhee and M. G. Kang, "Discrete cosine transform based regularized high-resolution image reconstruction algorithm," Optical Engineering, vol. 38, no. 8, pp. 1348-1356, 1999.

[12] R. R. Schultz and R. L. Stevenson, "Extraction of highresolution frames from video sequences," IEEE Transactions on Image Processing, vol. 5, no. 6, pp. 996-1011, 1996.

[13] H. Stark and P. Oskoui, "High-resolution image recovery from image-plane arrays, using convex projections," Journal of the Optical Society of America A, vol. 6, no. 11, pp. 1715-1726, 1989.

[14] A. J. Patti, M. I. Sezan, and A. Murat Tekalp, "Super-resolution video reconstruction with arbitrary sampling lattices and nonzero aperture time," IEEE Transactions on Image Processing, vol. 6, no. 8, pp. 1064-1076, 1997.

[15] R. Y. Tsai and T. S. Huang, "Multiframe image restoration and registration," in Advances in Computer Vision and Image Processing: Image Reconstruction from Incomplete Observations, T. S. Huang, Ed., vol. 1, chapter 7, pp. 317-339, JAI Press, Greenwich, Conn, USA, 1984.

[16] S. P. Kim and N. K. Bose, "Reconstruction of 2-D bandlimited discrete signals from nonuniform samples," IEE Proceedings. F, Radar and Signal Processing, vol. 137, no. 3, pp. 197-204, 1990.

[17] S. P. Kim and W.-Y. Su, "Recursive high-resolution reconstruction of blurred multiframe images," IEEE Transactions on Image Processing, vol. 2, no. 4, pp. 534-539, 1993.

[18] N. K. Bose, H. C. Kim, and H. M. Valenzuela, "Recursive total least squares algorithm for image reconstruction from noisy, undersampled frames," Multidimensional Systems and Signal Processing, vol. 4, no. 3, pp. 253-268, 1993.

[19] N. K. Bose, H. C. Kim, and B. Zhou, "Performance analysis of the TLS algorithm for image reconstruction from a sequence of undersampled noisy and blurred frames," in Proceedings of IEEE International Conference on Image Processing (ICIP '94), vol. 3, pp. 571-574, Austin, Tex, USA, November 1994.

[20] L. Poletto and P. Nicolosi, "Enhancing the spatial resolution of a two-dimensional discrete array detector," Optical Engineering, vol. 38, no. 10, pp. 1748-1757, 1999.

[21] P. J. B. Koeck, "Ins and outs of digital electron microscopy," Microscopy Research and Technique, vol. 49, no. 3, pp. 217-223, 2000.

[22] S. Peled and Y. Yeshurun, "Super-resolution in MRI: application to human white matter fiber tract visualization by diffusion tensor imaging," Magnetic Resonance in Medicine, vol. 45, no. 1, pp. 29-35, 2001.

[23] M. Elad and Y. Hel-Or, "A fast super-resolution reconstruction algorithm for pure translational motion and common spaceinvariant blur," IEEE Transactions on Image Processing, vol. 10, no. 8, pp. 1187-1193, 2001.

[24] S. Mann and R. W. Picard, "Video orbits of the projective group a simple approach to featureless estimation of parameters," IEEE Transactions on Image Processing, vol. 6, no. 9, pp. 1281-1295, 1997.

[25] S. Lertrattanapanich and N. K. Bose, "Latest results on highresolution reconstruction from video sequences," IEICE Tech. Rep. DSP99-140, The Institution of Electronic, Information and Communication Engineers, Tokyo, Japan, 1999, pp. 5965. 
[26] M. K. Ng, J. Koo, and N. K. Bose, "Constrained total leastsquares computations for high-resolution image reconstruction with multisensors," International Journal of Imaging Systems and Technology, vol. 12, no. 1, pp. 35-42, 2002.

[27] K. Sauer and J. Allebach, "Iterative reconstruction of bandlimited images from nonuniformly spaced samples," IEEE Transactions on Circuits and Systems, vol. 34, no. 12, pp. 1497-1506, 1987.

[28] H. Ur and D. Gross, "Improved resolution from subpixel shifted pictures," CVGIP: Graphical Models and Image Processing, vol. 54, no. 2, pp. 181-186, 1992.

[29] N. K. Bose and S. Lertrattanapanich, "Polynomial matrix factorization, multidimensional filter banks, and wavelets," in Sampling, Wavelets, and Tomography, J. J. Benedetto and A. I. Zayed, Eds., chapter 6, pp. 137-156, Birkhäuser, Boston, Mass, USA, 2004.

[30] S. Lertrattanapanich and N. K. Bose, "High resolution image formation from low resolution frames using Delaunay triangulation," IEEE Transactions on Image Processing, vol. 11, no. 12, pp. 1427-1441, 2002.

[31] N. Nguyen and P. Milanfar, "A wavelet-based interpolationrestoration method for super-resolution (Wavelet Superresolution)," Circuits Systems Signal Processing, vol. 19, no. 4, pp. 321-338, 2000.

[32] B. Bascle, A. Blake, and A. Zisserman, "Motion deblurring and super-resolution from an image sequence," in Proceedings of 4th European Conference on Computer Vision (ECCV '96), vol. 2, pp. 573-582, Springer, Cambridge, UK, April 1996.

[33] D. Rajan and S. Chaudhuri, "An MRF-based approach to generation of super-resolution images from blurred observations," Journal of Mathematical Imaging and Vision, vol. 16, no. 1 , pp. $5-15,2002$.

[34] G. H. Golub and C. F. Van Loan, Matrix Computations, Johns Hopkins University Press, Baltimore, Md, USA, 2nd edition, 1989.

[35] D. S. C. Biggs and M. Andrews, "Asymmetric iterative blind deconvolution of multiframe images," in Advanced Signal Processing Algorithms, Architectures, and Implementations VIII, vol. 3461 of Proceedings of SPIE, pp. 328-338, San Diego, Calif, USA, July 1998.

[36] M. B. Chappalli and N. K. Bose, "Enhanced Biggs-Andrews asymmetric iterative blind deconvolution," accepted for publication, August 2005, in Multidimensional Systems and Signal Processing and to appear in print in 2006.

[37] D. Rajan and S. Chaudhuri, "Simultaneous estimation of super-resolved scene and depth map from low resolution defocused observations," IEEE Transactions on Pattern Analysis and Machine Intelligence, vol. 25, no. 9, pp. 1102-1117, 2003.

[38] N. K. Bose and K. J. Boo, "Asymptotic eigenvalue distribution of block-Toeplitz matrices," IEEE Transactions on Information Theory, vol. 44, no. 2, pp. 858-861, 1998.

Nirmal K. Bose received the B. Tech. (with honors), M.S., and Ph.D. degrees in electrical engineering from the Indian Institute of Technology (IIT), Kharagpur, India; Cornell University, Ithaca, NY; and Syracuse University, Syracuse, NY, respectively. Currently, he is the HRB-Systems Professor of electrical engineering at the Pennsylvania State University, University Park. $\mathrm{He}$ is the author of Applied Multidimensional Systems Theory (Van Nostrand Reinhold, New York, 1982),

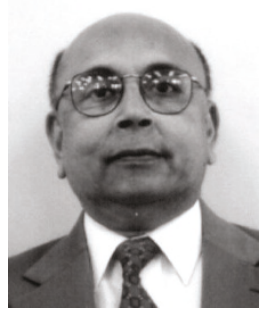

Digital Filters, Netherlands, 1985; Krieger, Malabar, (Elsevier, Amsterdam, The FL, 1993), the main author as well as the editor of Multidimensional Systems; Progress, Directions, and Open Problems (Reidel, Amsterdam, the Netherlands, 1985), coauthor of Neural Network Fundamentals with Graphs, Algorithms, and Applications (McGraw-Hill, New York, 1996) and the main author of Multidimensional Systems Theory and Applications (Kluwer, Dordrecht, 2003). He is the founding Editor-in-Chief of the International Journal on Multidimensional Systems and Signal Processing and serves on the editorial boards of several other journals. He has been a Fellow of the IEEE since 1981. He received several awards and honours including, most recently, the Fetter Endowment Award (20012004), the Alexander Von Humboldt Research Award from Germany in 2000 .

Michael K. Ng is a Professor of the Mathematics Department, Hong Kong Baptist University, and is an Adjunct Research Fellow in the E-Business Technology Institute at The University of Hong Kong. He was a Research Fellow (1995-1997) of Computer Sciences Laboratory, Australian National University, and an Assistant/Associate Professor (1997-2005) of the Mathematics Department, The University of Hong Kong, before joining Hong Kong Baptist University in 2005. He was one of the finalists and honourable mention of Householder Award IX, in 1996, at Switzerland, and he obtained an Excellent Young Researcher's Presentation at Nanjing International Conference on Optimization and Numerical Algebra, 1999. In 2001, he has been selected as one of the recipients of the Outstanding Young Researcher Award of The University of Hong Kong. He has published and edited several books, and published extensively in international journals and conferences, and has organized and served in many international conferences. Now, he serves on the editorial boards of SIAM Journal on Scientific Computing, Numerical Linear Algebra with Applications, Multidimensional Systems and Signal Processing, International Journal of Computational Science and Engineering, Numerical Mathematics, A journal of Chinese Universities (English Series), and several special issues of the international journals.

Andy C. Yau received the B.S. degree (19982001) from The Chinese University of Hong Kong, and the M.Phil degree (2002-2004) from The University of Hong Kong. He is a Ph.D. student at The University of Hong Kong. His research area is image processing and scientific computing.

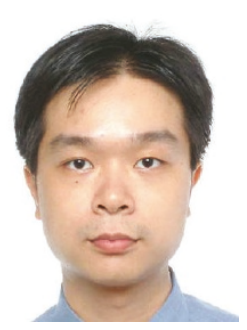

